PROCEEDINGS OF THE

AMERICAN MATHEMATICAL SOCIETY

Volume 140, Number 10, October 2012, Pages 3485-3493

S 0002-9939(2012)11229-8

Article electronically published on February 20, 2012

\title{
THE COMPLEMENTARY POLYNOMIALS AND THE RODRIGUES OPERATOR OF CLASSICAL ORTHOGONAL POLYNOMIALS
}

\author{
ROBERTO S. COSTAS-SANTOS AND FRANCISCO MARCELLÁN ESPAÑOL
}

(Communicated by Walter Van Assche)

\begin{abstract}
From the Rodrigues representation of polynomial eigenfunctions of a second order linear hypergeometric-type differential (difference or $q$-difference) operator, complementary polynomials for classical orthogonal polynomials are constructed using a straightforward method. Thus a generating function in a closed form is obtained.

For the complementary polynomials we present a second order linear hypergeometric-type differential (difference or $q$-difference) operator, a three-term recursion and Rodrigues formulas which extend the results obtained by $\mathrm{H}$. J. Weber for the standard derivative operator.
\end{abstract}

\section{INTRODUCTION}

Different authors (see [4, 5, 2, 17, 11] and the references therein) in various contexts dealt with Rodrigues' formula and generating functions.

In 4. Cryer studied the necessary conditions that we need to add to a system of polynomials satisfying a generalized Rodrigues formula for it to become a classical orthogonal system. In [17] Rasala studied the role of linear algebra in the theory of classical orthogonal polynomials, obtaining some general formulas. Later on, in [5. Hahn extended this result to $q$-polynomials by using the Hahn operator. In [2] Belmehdi gave an operator version of the Rodrigues formula concerning the classical orthogonal system and stated a characterization theorem for polynomials involved in such an expression.

On the other hand, in [1] Maroni and Van Iseghem characterized orthogonal families of polynomials whose generating function satisfies some first order linear partial differential equation.

We can write the (unnormalized) polynomial eigenfunctions $\left(p_{n}\right)$ of the second order linear differential (or difference or $q$-difference) operator $\mathscr{H}$ of hypergeometrictype, i.e.

$$
\mathscr{H} p_{n}(z)=\lambda_{n} p_{n}(z), \quad n \geq 0
$$

Received by the editors February 11, 2011 and, in revised form, April 8, 2011.

2010 Mathematics Subject Classification. Primary 33C45; Secondary 34B24, 42C05.

Key words and phrases. Classical orthogonal polynomials, Rodrigues operator, complementary polynomials, generating formula.

The first author acknowledges financial support from Dirección General de Investigación del Ministerio de Ciencia e Innovación of Spain under grant MTM2009-12740-C03-01 and from the program of postdoctoral grants (Programa de becas postdoctorales).

The second author acknowledges financial support from Dirección General de Investigación del Ministerio de Ciencia e Innovación of Spain under grant MTM 2009-12740-C03-01.

(C)2012 American Mathematical Society Reverts to public domain 28 years from publication 
by using the $n$-th Rodrigues operator associated with $(\phi, \mathbf{u}, z)$ where $\phi$ is a certain function and $\mathbf{u} \in \mathbb{P}^{\prime}$ is a classical linear functional (see [3]) as

$$
p_{n}(z)=\mathscr{R}_{n}(\phi, \mathbf{u}, z)(1), \quad n=0,1,2, \ldots .
$$

In $[3, \S 2, \S 3]$ the expressions of the operators $\mathscr{R}, \mathscr{H}$, and $\mathscr{D}$, as well as their polynomial eigenfunctions in the continuous, discrete and $q$-discrete case, respectively, are found.

Polynomial solutions of second order linear differential (or difference or $q$-difference) equations with coefficients depending on $n$ are studied in [8, 18, together with their orthogonality properties, which we therefore do not address here.

The aim of our contribution is to construct complementary polynomials of such eigenfunctions by using their Rodrigues operator representation (11) in a natural way extending the results of 19 for the standard derivative operator. The generating function of these complementary polynomials is obtained in a closed form allowing general properties shared by them.

The paper is organized as follows. In Section 2 we give the basic definitions and notation we use in the sequel. In Section 3 we introduce and construct the complementary polynomials in such a way that its Sturm-Liouville ordinary $\mathscr{D}$ equation is deduced. In Section 4 we establish their generating function (in the continuous case) and we show some identities that they satisfy. We finish this paper with some conclusions and outlooks.

\section{BASIC DEFINITIONS AND NOTATION}

In this section we will summarize the operational calculus background that we will use in the sequel. Since depending on the case the polynomials are orthogonal with respect to different linear functionals, we need to introduce some rigorous notation to embed all of them.

\subsection{Notation.}

(1) The lattice. For the general case we use the variable $z$. Notice that in the $q$-discrete case we need to replace $z$ by $x(s)=c_{1}(q) q^{s}+c_{2}(q) q^{-s}+c_{3}(q)$, where $q \in \widetilde{\mathbb{C}}:=\mathbb{C} \backslash\left[\{0\} \cup\left(\bigcup_{n \geq 1}\left\{z: z^{n}=1\right\}\right)\right]$ and $c_{1}, c_{2}$ and $c_{3}$ can depend on $q$ but not on $s$, and $z_{k}$ by $x_{k}(s)=x\left(s+\frac{k}{2}\right)$ for every $k \in \mathbb{Z}$. In the rest of the cases we replace $z$ and $z_{k}$ by $x$.

(2) The operators. For the general case we use some basic operators: $\mathscr{D}_{k}$, its dual $\mathscr{D}_{k}^{*}$, for every integer $k$, the identity $\mathscr{I}, \mathscr{E}^{+}$, and $\mathscr{E}^{-}$. So to recover the results in the continuous case (resp. discrete and $q$-discrete case) we need to replace $\mathscr{D}_{k}$ by $\frac{d}{d x}$ (resp. by $\Delta$, or by $\left.\frac{\Delta}{\Delta x(s+(k-1) / 2)}=\frac{\Delta}{\Delta x_{k-1}(s)}\right)$, and $\mathscr{E}^{+}$ by the identity $\mathscr{I}$ (resp. by the shift operator in both discrete cases) and $\mathscr{D} \equiv \mathscr{D}_{0}$.

Remark 2.1. The shift operator is usually denoted by $e^{\partial_{x}}$. Thus, in the discrete case we get $\left(e^{\partial_{x}} f\right)(x)=f(x+1)$, and in the $q$-discrete case $\left(e^{\partial_{x}} f\right)(x)=f(q x)$.

Notice that in the $q$-discrete case, $\mathscr{D}_{k}^{*}=\frac{\nabla}{\nabla x(s+k / 2)}$. Moreover, the forward and backward difference operators are defined as

$$
\begin{aligned}
& (\Delta f)(x):=\left(\mathscr{E}^{+}-\mathscr{I}\right)(f)(x)=f(x+1)-f(x), \\
& (\nabla f)(x):=\left(\mathscr{I}-\mathscr{E}^{-}\right)(f)(x)=f(x)-f(x-1) .
\end{aligned}
$$


(3) The constants. Throughout the paper we use some constants: $q, \alpha_{q}(n)$ and $[n]_{q}$. In the continuous and discrete cases for $q=1$, we set $\alpha_{q}(n)=1$ and $[n]=n$. In the $q$-discrete case we set $q \in \widetilde{\mathbb{C}}$ and

$$
\alpha_{q}(n)=\frac{q^{\frac{n}{2}}+q^{-\frac{n}{2}}}{2}, \quad[n]_{q}=\frac{q^{\frac{n}{2}}-q^{-\frac{n}{2}}}{q^{\frac{1}{2}}-q^{-\frac{1}{2}}} .
$$

2.2. Linear functionals. Let $\mathbb{P}$ be the linear space of polynomials with complex coefficients and let $\mathbb{P}^{\prime}$ be its dual space; i.e., $\mathbb{P}^{\prime}$ is the linear space of all linear functionals $\mathbf{u}: \mathbb{P} \rightarrow \mathbb{C}$. We denote by $\langle\mathbf{u}, p\rangle$ the duality bracket for $\mathbf{u} \in \mathbb{P}^{\prime}$ and $p \in \mathbb{P}$, and by $(\mathbf{u})_{n}=\left\langle\mathbf{u}, x^{n}\right\rangle$, with $n \geq 0$, the canonical moments of $\mathbf{u}$.

For any linear functional $\mathbf{u}$ and any polynomial $\pi$, let $\pi u, \mathscr{D}_{k}^{*} \mathbf{u}$, and $\mathscr{D}_{k} \mathbf{u}$ be the linear functionals defined by duality [6, 10,

$$
\begin{aligned}
\langle\pi \mathbf{u}, p\rangle & :=\langle\mathbf{u}, \pi p\rangle, \quad p \in \mathbb{P}, \\
\left\langle\mathscr{D}_{k}^{*} \mathbf{u}, p\right\rangle & :=-\left\langle\mathbf{u}, \mathscr{D}_{k} p\right\rangle, \quad p \in \mathbb{P}, \\
\left\langle\mathscr{D}_{k} \mathbf{u}, p\right\rangle & :=-\left\langle\mathbf{u}, \mathscr{D}_{k}^{*} p\right\rangle, \quad p \in \mathbb{P} .
\end{aligned}
$$

Taking into account these definitions we get

$$
\mathscr{D}(\pi \mathbf{u})=(\mathscr{M} \pi) \mathscr{D} \mathbf{u}+(\mathscr{D} \pi) \mathscr{M} \mathbf{u},
$$

where $\mathscr{M}$ is the forward mean arithmetic operator

$$
\mathscr{M}=\frac{1}{2}\left(\mathscr{E}^{+}+\mathscr{I}\right) .
$$

Furthermore, for two polynomials $\pi$ and $\xi$

$$
\mathscr{D}(\pi \xi)=\pi \mathscr{D} \xi+(\mathscr{D} \pi)\left(\mathscr{E}^{+} \xi\right)=(\mathscr{M} \pi) \mathscr{D} \xi+(\mathscr{D} \pi) \xi .
$$

The linear functional $\mathbf{u}$ is said to be a quasi-definite (or regular) linear functional if there exists a polynomial sequence $\left(p_{n}\right)$, with $\operatorname{deg} p_{n}=n$, which is orthogonal with respect to $\mathbf{u}$, i.e.

$$
\left\langle\mathbf{u}, p_{n} p_{m}\right\rangle=r_{n} \delta_{n, m}, \quad n, m \geq 0, \quad r_{n} \neq 0, \quad n \geq 0,
$$

where $\delta_{n, m}$ is the Kronecker symbol.

2.3. The classical orthogonal polynomials. Classical orthogonal polynomials, $\left(p_{n}\right)$, are the polynomial eigenfunctions of the second order linear differential (or difference or $q$-difference) operator of hypergeometric-type [16, 7]

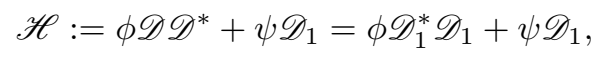

where $\hat{\phi}=\phi+\frac{1}{2} \psi \nabla z_{1}$ and $\psi$ are polynomials, with $\operatorname{deg} \hat{\phi} \leq 2$, $\operatorname{deg} \psi=1$. The corresponding eigenvalues are

$$
\lambda_{n}=[n]_{q}\left(\alpha_{q}(n-1) \psi^{\prime}+[n-1]_{q} \frac{\hat{\phi}^{\prime \prime}}{2}\right), \quad n \geq 0 .
$$

It is well known that such a family of polynomial eigenfunctions satisfies the following orthogonality conditions:

$$
\left\langle\mathbf{u}, p_{n} p_{m}\right\rangle=r_{n} \delta_{n, m}, \quad r_{n} \neq 0, \quad n, m \geq 0,
$$

where the linear functional $\mathbf{u}$ fulfills the distributional equation

$$
\mathscr{D}(\phi \mathbf{u})=\psi \mathbf{u} \text {. }
$$


As we said above, such polynomials can be represented in terms of the Rodrigues operator as follows:

$$
p_{n} \mathbf{u}=\mathscr{D}^{*<n>}\left(\mathbf{u}_{n}\right), \quad n \geq 0 .
$$

Here $\mathbf{u}_{k}:=\mathscr{E}^{+} \phi \mathbf{u}_{k-1}, k \geq 1$, with $\mathbf{u}_{0}:=\mathbf{u}$, and

$$
\mathscr{D}_{k}^{*<\ell>}:=\mathscr{D}_{k+1}^{*} \circ \mathscr{D}_{k+2}^{*} \circ \cdots \circ \mathscr{D}_{k+\ell}^{*} .
$$

Remark 2.2. Notice that although it is possible to find an integral representation for each classical linear functional $\mathbf{u}$, since the polynomial eigenfunctions of such a differential operator are the classical orthogonal polynomials (COP) [16], it is important to take into account that the techniques presented here can be applied in a more general framework for semiclassical orthogonal polynomials (see, for example, [9, 6, 12]).

2.4. The Rodrigues operator. The expression (6) for the polynomial eigenfunctions $p_{n}$ suggests considering the following operator.

Definition 2.1. Given a function $\phi$, a linear functional $\mathbf{u}$, and a lattice $z$, we define the $k$-th Rodrigues operator associated with $\left(\phi, \mathbf{u}_{m}, z_{m}\right)$, i.e.,

$$
\mathscr{R}_{k}\left(\phi, \mathbf{u}_{m}, z_{m}\right): \mathbb{P}_{n}\left[z_{m+k}\right] \longrightarrow \mathbb{P}_{n+1}\left[z_{m}\right]
$$

as

$$
\begin{gathered}
\mathscr{R}_{0}\left(\phi, \mathbf{u}_{m}, z_{m}\right):=\mathscr{I}, \quad \mathscr{R}_{1}\left(\phi, \mathbf{u}_{m}, z_{m}\right)(\bullet) \mathbf{u}_{m}:=\mathscr{D}_{m+1}^{*}\left(\bullet \mathbf{u}_{m+1}\right), \\
\mathscr{R}_{k}\left(\phi, \mathbf{u}_{m}, z_{m}\right):=\mathscr{R}_{1}\left(\phi, \mathbf{u}_{m}, z_{m}\right) \circ \mathscr{R}_{k-1}\left(\phi, \mathbf{u}_{m+1}, z_{m+1}\right), k \geq 2,
\end{gathered}
$$

which is well-defined according to the following result.

Lemma 2.1 ([3, Lemma 4.2]). If $(\phi, \mathbf{u}, z)$ is classical, then for any integer $k$ and any polynomial $\pi$, the function

$$
\mathscr{R}_{1}\left(\phi, \mathbf{u}_{k}, z_{k}\right)\left(\pi\left(z_{k+1}\right)\right)
$$

is a polynomial in $z_{k}$ of degree $\operatorname{deg}(\pi)+1$ with leading coefficient $\frac{\lambda_{m+2 k}}{[m+2 k]_{q}} \neq 0$, $m=\operatorname{deg} \pi$.

Remark 2.3. In the sequel, we set $\mathscr{R}_{k, \ell} \equiv \mathscr{R}_{k}\left(\phi, \mathbf{u}_{\ell}, z_{\ell}\right)$, with the convention $\mathscr{R}_{k}:=$ $\mathscr{R}_{k, 0}$. We also set $\psi_{k}(z):=\mathscr{R}_{1, k}(1)$, which satisfies the identity $\mathscr{D}_{k+1}^{*}\left(\mathbf{u}_{k+1}\right)=$ $\psi_{k} \mathbf{u}_{k}$.

\section{Complementary polynomials}

Let us now introduce the complementary polynomials, $\mathscr{P}_{\nu}(z ; n)$, which are defined in terms of the Rodrigues operator.

Definition 3.1 ([19]). Let $n, \nu$ be two nonnegative integers, with $\nu \leq n$. The complementary polynomial $\mathscr{P}_{\nu}(z ; n)$ of degree $\nu$ with respect to $p_{n}$ is defined through the relation

$$
p_{n}(z)=\mathscr{R}_{n-\nu}\left(\mathscr{P}_{\nu}(z ; n)\right) \text {. }
$$

Remark 3.1. Notice that these polynomials for $\nu=n-1$ were considered in [1, Theorems 2.1 and 2.2]. More generally, it is easy to check that $\mathscr{P}_{n-\nu}(z ; n)$ is, up to a constant, the $\nu$-th derivative (or difference or $q$-difference) of $p_{n}$, i.e. (see e.g. [14. (3.2.18), p. 66]),

$$
p_{n}^{(\nu)}=\mathscr{D}_{0}^{<\nu>} p_{n}:=\mathscr{D}_{\nu} \circ \mathscr{D}_{\nu-1} \circ \cdots \circ \mathscr{D}_{1}\left(p_{n}\right) .
$$


Taking into account the hypergeometric character of the $\mathscr{D}$-equation (4), the distributional equation (5) and Lemma 3.2 in [3], the following result is obtained in a straightforward way.

Theorem 3.1. The polynomials $\mathscr{P}_{\nu}(z ; n)$ satisfy the Rodrigues functional formulas

$\mathscr{P}_{\nu}(z ; n) \mathbf{u}_{n-\nu}=\mathscr{D}_{n-\nu}^{*<\nu-\mu>}\left(\mathscr{P}_{\mu}(z ; n) \mathbf{u}_{n-\mu}\right) \Longleftrightarrow \mathscr{P}_{\nu}(z ; n)=\mathscr{R}_{\nu-\mu, n-\nu} \circ \mathscr{R}_{\mu, n-\mu}(1)$.

Taking into account the last remark and the theory of COP, the following result holds (see also [19, Theorem 2.2] for standard derivative operator).

Lemma 3.1. $\mathscr{P}_{\nu}(z ; n)$ is a polynomial of degree $\nu$ satisfying the recurrence relation

$$
\mathscr{P}_{\nu+1}(z ; n)=\phi\left(\mathscr{D}_{n-\nu}^{*} \mathscr{P}_{\nu}(z ; n)\right)+\psi_{n-\nu-1} \mathscr{P}_{\nu}(z ; n) \text {. }
$$

Notice that from the Rodrigues operator formula (6), $\mathscr{P}_{0}(z ; n) \equiv 1$.

Proof. We will prove (10) by induction.

Case $\nu=1$ is deduced by carrying out explicitly from (6), which is a natural way of working with the Rodrigues operator formula (6). It yields

$$
p_{n}(z) \mathbf{u}=\mathscr{D}^{*<n-1>}\left(\psi_{n-1} \mathbf{u}_{n-1}\right) .
$$

By Definition 3.1 we get

$$
\mathscr{P}_{1}(z ; n)=\psi_{n-1}(z)=\mathscr{D}_{z-1} \phi+\mathscr{D}_{z-1}\left(z_{n-1}\right)\left(\mathscr{E}^{+} \psi_{n-2}\right) .
$$

Assuming the validity of (7) for $\nu$ we get

$$
\begin{aligned}
p_{n}(z) \mathbf{u} & =\mathscr{D}^{*<n-\nu-1>}\left(\mathscr{D}_{n-\nu}^{*}\left(\mathscr{P}_{\nu}(z ; n) \mathbf{u}_{n-\nu}\right)\right) \\
& =\mathscr{D}^{*<n-\nu-1>}\left(\left(\mathscr{D}_{n-\nu}^{*} \mathscr{P}_{\nu}(z ; n)\right)\left(\mathscr{E}^{-} \mathbf{u}_{\mathbf{n}-\nu}\right)+\mathscr{P}_{\nu}(z ; n) \psi_{n-\nu-1} \mathbf{u}_{\mathbf{n}-\nu-\mathbf{1}}\right) \\
(12) \quad & =\mathscr{D}^{*<n-\nu-1>}\left(\left\{\phi\left(\mathscr{D}_{n-\nu}^{*} \mathscr{P}_{\nu}(z ; n)\right)+\psi_{n-\nu-1} \mathscr{P}_{\nu}(z ; n)\right\} \mathbf{u}_{\mathbf{n}-\nu-\mathbf{1}}\right) .
\end{aligned}
$$

Taking into account

$$
p_{n}(z) \mathbf{u}=\mathscr{D}^{*<n-\nu-1>}\left(\mathscr{P}_{\nu+1}(z ; n) \mathbf{u}_{n-\nu-1}\right)
$$

and the comparison with the right-hand side of (12), (17) follows by induction together with the recursive equation (10), which yield constructing systematically the complementary polynomials starting from $\mathscr{P}_{0}(z ; n) \equiv 1$ in a constructive way.

Remark 3.2. By using (11) and a straightforward computation we get [14]

$$
\psi_{n}=\frac{\mathscr{E}^{+n}\left(\phi+\psi \Delta z_{-1}\right)-\phi}{\Delta z_{n-1}} .
$$

Furthermore, the polynomial $\mathscr{D}_{0}^{<k>} p_{n}$ is an eigenfunction of the linear operator

$$
\mathscr{H}_{k}:=\phi \mathscr{D}_{k} \mathscr{D}_{k}^{*}+\psi_{k} \mathscr{D}_{k+1}=\phi \mathscr{D}_{k+1}^{*} \mathscr{D}_{k+1}+\psi_{k} \mathscr{D}_{k+1} \text {. }
$$

Its corresponding eigenvalue is

$$
\mu_{n, k}=\lambda_{n}+\sum_{m=0}^{n-1} \mathscr{D}_{m+1} \psi_{m}=\frac{[n-k]_{q}}{[n+k]_{q}} \lambda_{n+k}
$$


3.1. The Sturm-Liouville ordinary $\mathscr{D}$-equation. If we take into account Remark 3.2, which reflects the hypergeometric character of the operator $\mathscr{H}$, as well as Remark 3.1 and Theorem 3.1] we obtain the following result in a straightforward way (see also [19, Theorem 3.2] for standard derivative operator).

Theorem 3.2. The polynomials $\mathscr{P}_{\nu}(x ; n)$ satisfy the Sturm-Liouville ordinary $\mathscr{D}$ equation

$$
\mathscr{D}_{n-\nu+1}^{*}\left(\left(\mathscr{D}_{n-\nu+1} \mathscr{P}_{\nu}(z ; n)\right) \mathbf{u}_{n-\nu+1}\right)=\mu_{n, n-\nu} \mathscr{P}_{\nu}(z ; n) \mathbf{u}_{n-\nu} .
$$

Assuming $\mathbf{u}$ regular, the above expression leads to

$$
\begin{aligned}
\mathscr{H}_{n-\nu} \mathscr{P}_{\nu}(z ; n) & =\phi \mathscr{D}_{n-\nu+1}^{*}\left(\mathscr{D}_{n-\nu+1} \mathscr{P}_{\nu}(z ; n)\right)+\psi_{n-\nu}\left(\mathscr{D}_{n-\nu+1} \mathscr{P}_{\nu}(z ; n)\right) \\
& =\mu_{n, n-\nu} \mathscr{P}_{\nu}(z ; n) .
\end{aligned}
$$

\section{Generating function}

In this section we focus our attention on the continuous case, i.e., $\mathscr{D}_{k}^{*}=\mathscr{D}_{k}=$ $\frac{d}{d x}$, and $z_{k}=z=x$ for every integer $k$, taking into account the difficulties of computations in the general case.

Definition 4.1. The generating function for the polynomials $\mathscr{P}_{\nu}(x ; n)$ is

$$
\mathscr{P}(y, x ; n)=\sum_{\nu=0}^{\infty} \frac{y^{\nu}}{\nu !} \mathscr{P}_{\nu}(x ; n) .
$$

The series converges for $|y|<\epsilon$ for some $\epsilon>0$. It can be given in a closed form assuming the generating function is regular at $x$.

Theorem 4.1. The generating function for the polynomials $\mathscr{P}_{\nu}(x ; n)$ is given by

$$
\begin{gathered}
\mathscr{P}(y, x ; n) \mathbf{u}=\left(\frac{\phi(x+y \phi(x))}{\phi(x)}\right)^{n} \widetilde{\mathbf{u}} \\
\frac{\partial^{\mu}}{\partial y^{\mu}} \mathscr{P}(y, x ; n) \mathbf{u}=\left(\frac{\phi(x+y \phi(x))}{\phi(x)}\right)^{n-\mu} \mathscr{P}_{\mu}(x+y \phi(x) ; n) \widetilde{\mathbf{u}},
\end{gathered}
$$

where $\widetilde{\mathbf{u}}=\exp \left(y \phi(x) \frac{d}{d x}\right) \circ \mathbf{u}$.

Proof. Taking into account that $\mathbf{u}_{k}=\phi^{k} \mathbf{u}$, (14) follows by multiplying the generating function by $\phi^{n}$ and replacing the Rodrigues operator formula, (8), in (13). Indeed,

$\phi^{n}(x) \mathscr{P}(y, x ; n) \mathbf{u}=\mathscr{P}(y, x ; n) \mathbf{u}_{n}=\sum_{\nu=0}^{\infty} \frac{y^{\nu}}{\nu !} \mathscr{P}_{\nu}(x ; n) \phi^{n}(x) \mathbf{u}=\sum_{\nu=0}^{\infty} \frac{(y \phi(x))^{\nu}}{\nu !} \frac{d^{\nu}}{d x^{\nu}} \mathbf{u}_{n}$,

which converges for $|y \phi(x)|<\epsilon$ for a suitable $\epsilon>0$ if $x$ is a regular point of the generating function.

In fact, at this point, the series can be summed exactly, because the expression inside the derivatives is independent of the summation. Thus we obtain

$$
\phi^{n}(x) \mathscr{P}(y, x ; n) \mathbf{u}=\exp \left(y \phi(x) \frac{d}{d x}\right)\left(\phi^{n}(x) \mathbf{u}\right)=\phi^{n}(x+y \phi(x)) \widetilde{\mathbf{u}},
$$

and, therefore,

$$
\mathscr{P}(y, x ; n) \mathbf{u}=\left(\frac{\phi(x+y \phi(x))}{\phi(x)}\right)^{n} \widetilde{\mathbf{u}}
$$


Differentiating (13) and substituting here the generalized Rodrigues formula (9), we get (15) in a similar way.

Remark 4.1. If the linear functional $\mathbf{u}$ has an integral representation

$$
\langle\mathbf{u}, \pi\rangle=\int_{\Omega} \pi(z) \rho(z) d z
$$

where $\Omega$ is a contour in the complex plane, then we can write (17) as

$$
\mathscr{P}(y, x ; n)=\left(\frac{\phi(x+y \phi(x))}{\phi(x)}\right)^{n} \frac{\rho(x+y \phi(x))}{\rho(x)} .
$$

Since we consider recurrence relations, the case $\mu=1$ of (15) can be read in terms of partial differential equations (PDEs) (see also [19, Theorem 3.3] for the standard derivative operator).

Theorem 4.2. The generating function satisfies the PDEs

$$
\begin{aligned}
& \left(1+y \phi^{\prime}(x)+\frac{1}{2} y^{2} \phi^{\prime \prime} \phi(x)\right) \frac{\partial \mathscr{P}(y, x ; n)}{\partial y}=\left(\mathscr{P}_{1}(x ; n)+y \phi(x) \mathscr{P}_{1}^{\prime}(x ; n)\right) \mathscr{P}(y, x ; n), \\
& \left(1+y \phi^{\prime}(x)+\frac{1}{2} y^{2} \phi^{\prime \prime} \phi(x)\right) \frac{\partial \mathscr{P}(y, x ; n)}{\partial x}=\left\{\left(1+y \phi^{\prime}(x)\right) \mathscr{P}_{1}^{\prime}(x ; n)-\frac{1}{2} y \phi^{\prime \prime} \mathscr{P}_{1}(x ; n)\right\} \\
& \phi(x) \frac{\partial \mathscr{P}(y, x ; n)}{\partial x}=\left(1+y \phi^{\prime}(x)\right)\left(\psi(x)+(n-1) \phi^{\prime}(x)+y \phi(x)\left(\psi^{\prime}+(n-1) \phi^{\prime \prime}\right)\right) \\
& \times \mathscr{P}(y, x ; n-1)-\left(\psi(x)+(n-1) \phi^{\prime}(x)\right) \mathscr{P}(y, x ; n) .
\end{aligned}
$$

Proof. From (15) for $\mu=1$, (14), and taking into account that $\mathbf{u}$ is regular we obtain

$$
\phi(x+y \phi(x)) \frac{\partial \mathscr{P}(y, x ; n)}{\partial y}=\phi(x)\left(\psi(x+y \phi(x))+(n-1) \phi^{\prime}(x+y \phi(x))\right) \mathscr{P}(y, x ; n) .
$$

Substituting in (22) the Taylor series-type expansions

$$
\begin{aligned}
\phi(x+y \phi(x)) & =\phi(x)\left(1+y \phi^{\prime}(x)+\frac{1}{2} y^{2} \phi^{\prime \prime} \phi(x)\right), \\
\phi^{\prime}(x+y \phi(x)) & =\phi^{\prime}(x)+y \phi^{\prime \prime} \phi(x), \\
\psi(x+y \phi(x)) & =\psi(x)+y \psi^{\prime}(x) \phi(x),
\end{aligned}
$$

since $\phi$ and $\psi$ are polynomials of degree at most 2 and 1 , respectively, we get (18). Using $n-1$ instead of $n$ in the generating function we obtain (19) (see also (28) in [19]).

Differentiating (16) with respect to $x$ yields (21). Using the exponent $n$ instead of $n-1$ of the generating function together with (21) we get (20), where we assume the linear functional is regular.

In fact the polynomials $\mathscr{P}_{\nu}(x ; n)$ satisfy different recursions and other relations which are the same as those H. J. Weber obtained in [19, §4] and, as a consequence, we omit them here. 


\section{Conclusions And OUtLOOKS}

We have used a natural way to deal with the Rodrigues operator formula for the polynomial eigenfunctions of the second order linear differential (difference or $q$-difference) operator (5) which leads to a family of closely related complementary polynomials that satisfy the Rodrigues formulas. Their generating function (in the continuous case) is obtained in a closed form.

The discrete and $q$-discrete versions for the generating function will be studied in a work in progress.

Furthermore, since the $\mathscr{D}$-equation (5) is of hypergeometric-type, the complementary polynomials satisfy an analogous $\mathscr{D}$-equation (see Theorem 3.2 ). Indeed, a straightforward calculation shows that this method generates all the basics of COP, discrete COP, and $q-\mathrm{COP}$ (see, for example, [3, 13, 15] and the references therein).

Moreover, this method, also considered in a similar way by H. J. Weber, is not restricted to COP. In fact we believe it can be applied to semiclassical orthogonal polynomials and even in a more general framework.

\section{ACKNOWLEDGEMENT}

The authors thank the referee for suggestions and comments which have contributed to improving the presentation of the manuscript.

\section{REFERENCES}

1. M. Alfaro and R. Álvarez-Nodarse. A characterization of the classical orthogonal discrete and q-polynomials. J. Comput. Appl. Math. 201 (2007), 48-54. MR.2293537 (2008h:33013)

2. S. Belmehdi. Rodrigues's formula revisited: a magic array. Personal communication.

3. R. S. Costas-Santos and F. Marcellan. $q$-Classical orthogonal polynomials: A general difference calculus approach. Acta Appl. Math. 111 (2010), 107-128. MR.2653053 (2011g:33012)

4. C. W. Cryer. Rodrigues' formula and the classical orthogonal polynomials. Boll. Un. Mat. Ital. (4) 3 (1970), 1-11. MR0259197 (41:3839)

5. W. Hahn. On the paper: "The Rodrigues formula and polynomial differential operators". J. Math. Anal. Appl. 96 (1983), 52-53. MR7717493(85f:33004)

6. L. Kheriji. An introduction to the $H_{q}$-semiclassical orthogonal polynomials. Meth. Appl. Anal. 10 (2003), 387-412. MR2059943 (2005m:33034)

7. R. Koekoek, P. A. Lesky, R. F. Swarttouw. Hypergeometric Orthogonal Polynomials and Their q-Analogues, Springer Monographs in Mathematics, Springer-Verlag, Berlin, 2010. MR.2656096 (2011e:33029)

8. P. A. Lesky. Endliche und unendliche Systeme von kontinuierlichen klassischen Orthogonalpolynomen. Z. Angew. Math. Mech. 76 (1996), 181-184. MR.1382858 (97e:33009)

9. P. Maroni, Une Théorie Algebrique des Polynômes Orthogonaux: Applications aux Polynômes Orthogonaux Semi-classiques, in: C. Brezinski et al. (eds.), Orthogonal Polynomials and Their Applications, IMACS Annals on Comput. and Appl. Math. 9, J.C. Baltzer AG, Basel, 1991, 98-130. MR.1270222 (95i:42018)

10. P. Maroni. Semiclassical character and finite-type relations between polynomial sequences. Appl. Num. Math. 31 (1999), 295-330. MR1711161 (2001f:42038)

11. P. Maroni and J. Van Iseghem. Generating functions and semi-classical orthogonal polynomials. Proc. Roy. Soc. Edinburgh Sect. A 124 (1994), 1003-1011. MR.1303766 (96a:33011)

12. J. C. Medem. A family of singular semi-classical functionals. Indag. Mathem. (N.S.) 13 (2002), 351-362. MR2057053 (2005d:42035)

13. J. C. Medem, R. Álvarez-Nodarse, and F. Marcellán. On the $q$-polynomials: a distributional study. J. Comput. Appl. Math. 135 (2001), 157-196. MR.1850540(2003f:33027)

14. A. F. Nikiforov, S. K. Suslov, and V. B. Uvarov. Classical Orthogonal Polynomials of a Discrete Variable. Springer Series in Computational Physics. Springer-Verlag, Berlin, 1991. MR.1149380 (92m:33019) 
15. A. F. Nikiforov and V. B. Uvarov. Classical orthogonal polynomials in a discrete variable on nonuniform lattices. Inst. Prikl. Mat. Im. M. V. Keldysha Akad. Nauk SSSR, 17, 1983. MR 0753537 (86c:39006)

16. A. F. Nikiforov and V. B. Uvarov. The Special Functions of Mathematical Physics. Birkhäuser Verlag, Basel, 1988. MR 922041 (89h:33001)

17. R. Rasala. The Rodrigues formula and polynomial differential operators. J. Math. Anal. Appl. 84 (1981), 443-482. MR639676 (83g:33009)

18. A. Ronveaux, A. Zarzo, I. Area and E. Godoy. Classical orthogonal polynomials: dependence of parameters. J. Comput. Appl. Math. 121 (2000), 95-112. MR.1780044(2002a:33016)

19. H. J. Weber. Connections between real polynomial solutions of hypergeometric-type differential equations with Rodrigues formula. Cent. Eur. J. Math. 5 (2007), 415-427. MR2301942 (2008e:33023)

Departamento de Matemáticas, Facultad de Ciencias, Universidad de Alcalá, 28871 Alcalá de Henares, Spain

E-mail address: rscosa@gmail.com

E-mail address: roberto.costas@uah.es

Departamento de Matemáticas, Universidad Carlos iti de Madrid, Avenida de la UNIVERSIDAD 30, 28911 LEgANÉS, SPAIN

E-mail address: pacomarc@ing.uc3m.es 